\title{
Consumo de alimentos en comederos ambulantes como factor de riesgo para síntomas dispépticos en estudiantes de medicina
}

\section{Street food consumption as a risk factor for symptoms of dyspepsia in medical students}

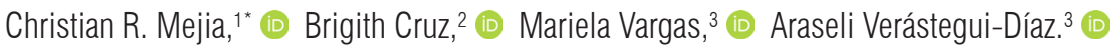

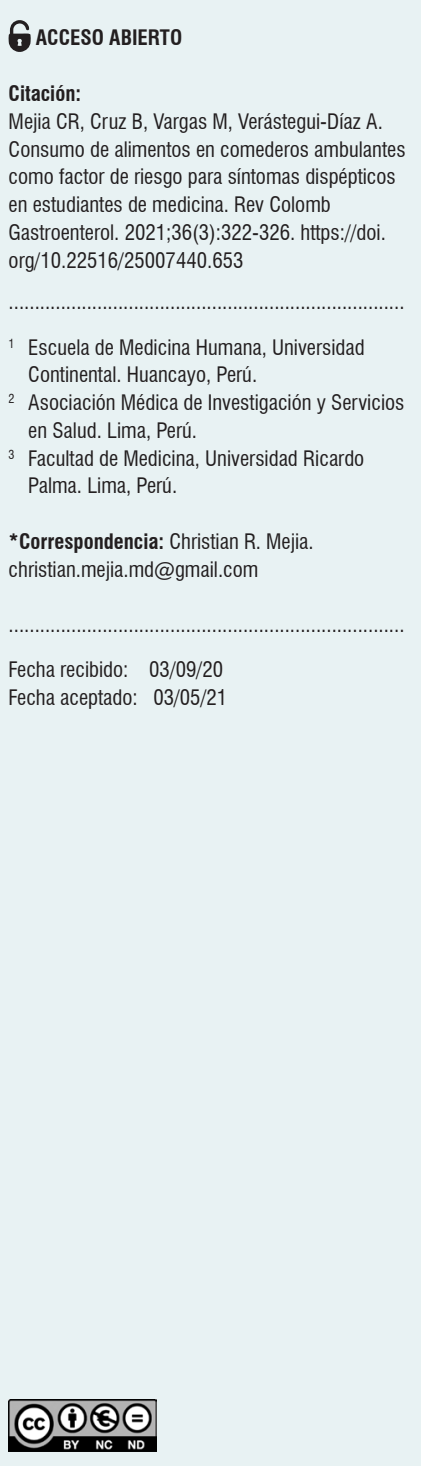

\begin{abstract}
Resumen
Introducción: los estudiantes universitarios muchas veces realizan su alimentación en comederos ambulantes, lo que puede ser causa de sintomatología digestiva, pero esto no se ha evidenciado en estudios en dicha población. Objetivo: determinar si el consumo de alimentos en comederos ambulantes está asociado con síntomas dispépticos en los estudiantes de medicina peruanos. Material y métodos: se realizó un estudio multicéntrico de datos secundarios, del encuestado de 1797 estudiantes de medicina en ocho facultades de medicina, se calculó una potencia estadística del $93 \%$. El padecimiento de síntomas dispépticos se asoció con el antecedente de consumo de alimentos en comederos ambulantes. Se obtuvo estadísticas de asociación con modelos bivariados y multivariados. Resultados: El rango de dispepsia funcional varió entre el $9 \%$ y el $28 \%$ y el de consumo de alimentos en comederos ambulantes, entre $1 \%$ y $5 \%$. En el análisis multivariado, consumir alimentos en comederos ambulantes era un factor asociado con la mayor frecuencia de padecer dispepsia funcional (razón de prevalencia ajustada [RPa]: 1,45; intervalo de confianza [IC] $95 \%$ : 1,09-1,94; $p$ $=0,010$ ); además, otras variables que resultaron significativas fueron el sexo femenino (RPa: 1,40; IC $95 \%$ : $1,15-1,71 ; p=0,001$ ) y los que comían en horarios similares (RPa: 0,76; IC $95 \%: 0,61-0,94 ; p=0,012$ ), ajustadas por la edad y el semestre académico que cursaban. Conclusiones: Los estudiantes que consumían sus alimentos en comederos ambulantes tenían mayor frecuencia de padecer síntomas dispépticos, esto debe ser vigilado por las autoridades sanitarias y universitarias, ya que puede generar repercusiones a corto y largo plazo.
\end{abstract}

\section{Palabras clave (DeCS)}

Dispepsia funcional, estudiantes de medicina, Perú.

\begin{abstract}
Introduction: University students often eat at street food stalls, which can cause various digestive symptoms, although this has not been evidenced in studies carried out in this population. Objective: To establish whether food consumption in street food stalls is associated with symptoms of dyspepsia in Peruvian medical students. Materials and methods: A secondary data analysis of a multicenter study was conducted. Of 1797 medical students surveyed in eight medical schools, a statistical power of $93 \%$ was calculated. Symptoms of dyspepsia were associated with a history of food consumption in street food stalls. Association statistics were obtained with bivariate and multivariate models. Results: The prevalence of functional dyspepsia varied between $9-28 \%$ and food consumption in street stalls between $1-5 \%$. In the multivariate analysis, eating food in the street was a factor associated with a greater frequency of suffering from functional dyspepsia (aPR: 1.45; $95 \% \mathrm{Cl}: 1.09-1.94 ; \mathrm{p}=0.010)$. In addition, other significant variables were the female sex (aPR: $1.40 ; 95 \% \mathrm{Cl}$ : $1.15-1.71 p=0.001$ ) and people eating at similar times (aPR: 0.76; $95 \% \mathrm{Cl}: 0.61-0.94 ; p=0.012$ ) adjusted for age, and academic term. Conclusions: Dyspepsia symptoms were more common in students who ate their food from street stalls. This should be monitored by health and university authorities, as it can have short- and long-term consequences.
\end{abstract}

\section{Keywords}

Functional dyspepsia; Medical students; Peru. (Source: DeCS) 


\section{INTRODUCCIÓN}

Las enfermedades gástricas siguen estando dentro de las principales causas de enfermedades agudas y crónicas de la población en general ${ }^{(1)}$. Diversos estudios que evalúan los factores que influyen en su aparición han demostrado que la calidad de la alimentación es un factor trascendental, que junto con otros factores relacionados propician el desarrolo de enfermedades gastrointestinales ${ }^{(2,3)}$, especialmente en países de Latinoamérica (como Perú), donde la higiene de la preparación, conservación y expendio de alimentos es aún deficiente ${ }^{(4)}$, situación que incrementa el riesgo de adquirir enfermedades del tracto gastrointestinal de tipo infeccioso y parasitario ${ }^{(5-7)}$.

Esta situación cobra mayor importancia en poblaciones como la estudiantil universitaria, que debido al limitado tiempo con que cuentan, escasos recursos económicos y poco cuidado de su salud individual, buscan adquirir estos alimentos de la manera más sencilla y rápida, lo que los expone a un mayor riesgo de adquirir enfermedades infecciosas gastrointestinales. Estudios recientes encontraron que entre el $24 \%$ y el $46 \%$ de los estudiantes de medicina de países de Latinoamericana padecía de dispepsia funcional, asociado con diversos factores sociales, educativos y otros hábitos ${ }^{(4)}$. Pero dichos estudios no se enfocaron en la manera en que los alumnos consiguen sus alimentos, por lo que el objetivo de esta investigación fue determinar si el consumo de alimentos expendidos por vendedores ambulantes está asociado con síntomas dispépticos en los estudiantes de medicina peruanos.

\section{MATERIAL Y MÉTODOS}

Se realizó un estudio multicéntrico de análisis de datos secundarios, que se basó en una muestra obtenida del estudio primario realizado en 2016 para la determinación de los factores que se asociaron a la dispepsia funcional entre los estudiantes de medicina ${ }^{(4)}$. En el estudio principal se realizó una encuesta a los estudiantes de medicina y se incluyó la data de aquellos estudiantes que respondieron satisfactoriamente las preguntas para determinar si padecían dispepsia y el lugar donde ingerían sus alimentos, se excluyó a un estudiante por haber sido diagnosticado de dispepsia orgánica, tres por haber tenido úlcera péptica, seis por enfermedad de reflujo gastroesofágico y 116 por haber sido diagnosticados de gastritis. La población final fue de 1797 estudiantes de siete departamentos del Perú: 400 de Lima, 539 de Piura, 220 de Cajamarca, 277 de Huancayo, 109 de Cusco, 101 de Ica y 151 de Ucayali.

La variable que mostraba el padecimiento de síntomas dispépticos fue medida mediante una prueba validada en la población peruana en la cual se pregunta acerca del padecimiento de sintomatología en nueve escenarios: fácil sensación de plenitud (la sensación de estar lleno antes de lo normal), plenitud epigástrica posprandial (la sensación de estar lleno después de comer menos de lo usual), pirosis (la sensación de quemadura que sube desde el estómago hasta la faringe), regurgitación (el regreso sin esfuerzo del contenido alimentario a través del esófago), náuseas (la sensación de tener ganas de vomitar), vómitos (la expulsión violenta y espasmódica del contenido del estómago a través de la boca), dolor epigástrico posprandial (la sensación molesta en la boca del estómago o el epigastrio de forma continua o intermitente después de ingerir alimentos), eructos excesivos (la expulsión del exceso de aire del tracto digestivo superior que sobrepasa la normalidad) y dolor del hambre (la sensación de vacío estomacal, esencialmente en el abdomen). Se consideraba positivo a los que tuvieran tres o más síntomas con una intensidad moderada o muy frecuente, la prueba que se usó para esto tenía un $95 \%$ de sensibilidad y un $100 \%$ de especificidad ${ }^{(8)}$. La variable independiente principal fue el lugar donde regularmente ingerían sus alimentos, y la categoría de interés era si lo realizaban en sitios de ventas ambulantes. Además, se consideraron las variables de ajuste: sexo (masculino/femenino), edad (tomada de forma cuantitativa para la estadística descriptiva y analítica), semestre de estudio (tomada de forma cuantitativa para la estadística descriptiva y analítica) y comer en horarios similares (sí/no).

Luego de adquirir la base de datos para el análisis secundario, se procedió a la depuración de los mismos con los criterios de selección, que fue realizado en el programa Microsoft Excel 2010 (versión para Windows). Por último, se trasladaron los datos al programa Stata 11,1 (StataCorp LP, College Station, TX, Estados Unidos); en el cual se realizaron todos los análisis estadísticos.

Para el análisis descriptivo, las variables cualitativas fueron analizadas con sus frecuencias y porcentajes. Las variables cuantitativas se describieron con la mediana y rango intercuartílico (RIC), previa confirmación de su no normalidad con la prueba de Shapiro-Wilk. Para la estadística analítica se usó la prueba de chi cuadrado para el cruce de dos variables cualitativas y la suma de rangos para el cruce de una cualitativa frente a una cuantitativa. Por último, se desarrolló un análisis mediante modelos lineales generalizados usando la familia Poisson, la función de enlace log y modelos robustos, y se consideró a la universidad sede como grupo clúster (para tomar en cuenta la individualidad de cada población evaluada); con esto se obtuvieron las razones de prevalencias crudas $(\mathrm{RPc})$ y ajustadas $(\mathrm{RPa})$, con sus intervalos de confianza del $95 \%$ (IC $95 \%$ ) y los valores $p$. Se consideraron estadísticamente significativos los valores $p<0,05$. 


\section{RESULTADOS}

De los 1797 estudiantes de medicina, el $53 \%$ (960) fueron mujeres, la mediana de edades fue 20 años (RIC: 18-22 años de edad). El $3 \%$ (52) manifestó que comía regularmente en sitios de ventas ambulantes y el $23 \%$ (412) tuvo un diagnóstico positivo para dispepsia. $\mathrm{Al}$ realizar la descripción de la población según el comer en ventas ambulantes se encontró similitudes en las frecuencias del sexo, las edades y semestres; sin embargo, hubo diferencias según el comer en horarios similares $(p=0,021)$ (Tabla $\mathbf{1})$.

Tabla 1. Características de los estudiantes de medicina según el consumo de alimentos en sitios de ventas ambulantes

\begin{tabular}{|c|c|c|c|}
\hline \multirow[t]{2}{*}{ Variables } & \multicolumn{2}{|c|}{ Come en ventas ambulantes $n(\%)$} & \multirow[t]{2}{*}{ Valor $p$} \\
\hline & Sí & No & \\
\hline \multicolumn{4}{|l|}{ Sexo } \\
\hline - Femenino & $25(48,1)$ & $929(53,5)$ & 0,441 \\
\hline - Masculino & $27(51,9)$ & $808(46,5)$ & \\
\hline Edad (años)* & $20(19-23)$ & $20(18-22)$ & 0,105 \\
\hline Semestre* $^{*}$ & $4(3-7,5)$ & $5(2-8)$ & 0,655 \\
\hline \multicolumn{4}{|c|}{ Come en horarios similares } \\
\hline - Sí & $20(38,5)$ & $782(45,4)$ & 0,021 \\
\hline - No & $32(61,5)$ & $941(54,6)$ & \\
\hline
\end{tabular}

*Mediana y RIC. Valores $p$ obtenidos con la prueba de chi cuadrado para el cruce de dos variables cualitativas y con la suma de rangos para el cruce de una cualitativa frente a una cuantitativa.
En la Figura 1 se puede observar que el rango de dispepsia funcional varió entre $9 \%$ y $28 \%$, y el de consumo de alimentos en ventas ambulantes, entre $1 \%$ y $5 \%$.

En la Figura 2 se puede observar que los porcentajes de consumo de alimentos fueron similares según si se tenía (4\%) o no dispepsia (3\%), y esto fue estadísticamente similar.

$\mathrm{Al}$ realizar el análisis multivariado se encontró que consumir alimentos en ventas ambulantes era un factor asociado con la mayor frecuencia de padecer dispepsia funcional (RPa: 1,45; IC 95 \%: 1,09-1,94; $p=0,010)$. Además, otras variables que resultaron significativas fueron el sexo femenino (RPa: 1,40; IC $95 \%$ : 1,15-1,71; $p=0,001$ ) y los que comían en horarios similares (RPa: 0,76; IC $95 \%$ : $0,61-0,94 ; p=0,012)$, todas estas ajustadas por la edad y el semestre académico que cursaban (Tabla 2).

\section{DISCUSIÓN}

Se encontró que un pequeño porcentaje de los estudiantes de medicina comía regularmente en sitios de ventas ambulantes, pero esto fue un factor asociado con el padecimiento de dispepsia funcional; a pesar de que no se encontraron estudios sobre la posible asociación, Barker y colaboradores identificaron el riesgo de padecimiento de enfermedades gastrointestinales y el consumo de alimentos expendidos por vendedores ambulantes ${ }^{(9)}$, posiblemente debido a que los alimentos que comercian estos establecimientos tienen muchos problemas en la salubridad, como se ha reportado en diversos estudios internacionales, donde se identifican valores inaceptables de contaminantes microbiológicos ${ }^{(10-13)}$.

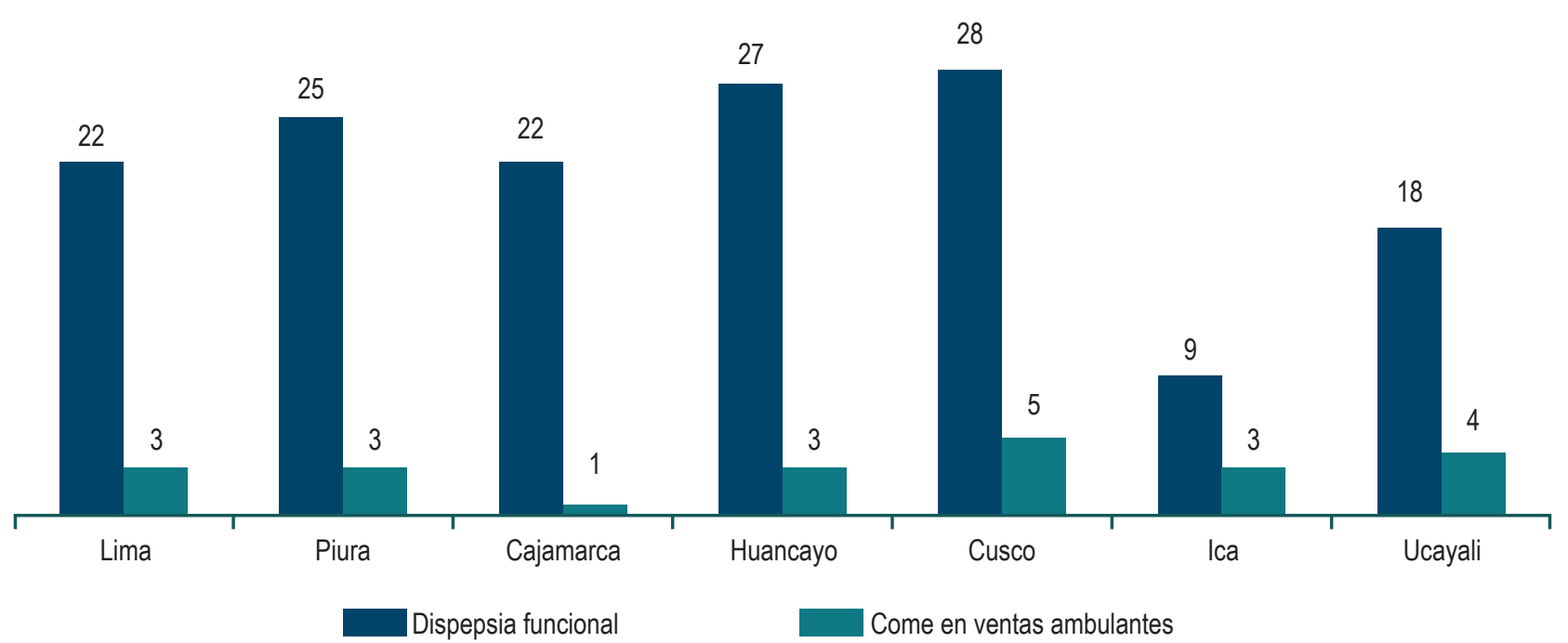

Figura 1. Porcentaje de dispepsia funcional y consumo de alimentos en sitios de ventas ambulantes en estudiantes de medicina de siete departamentos del Perú. 


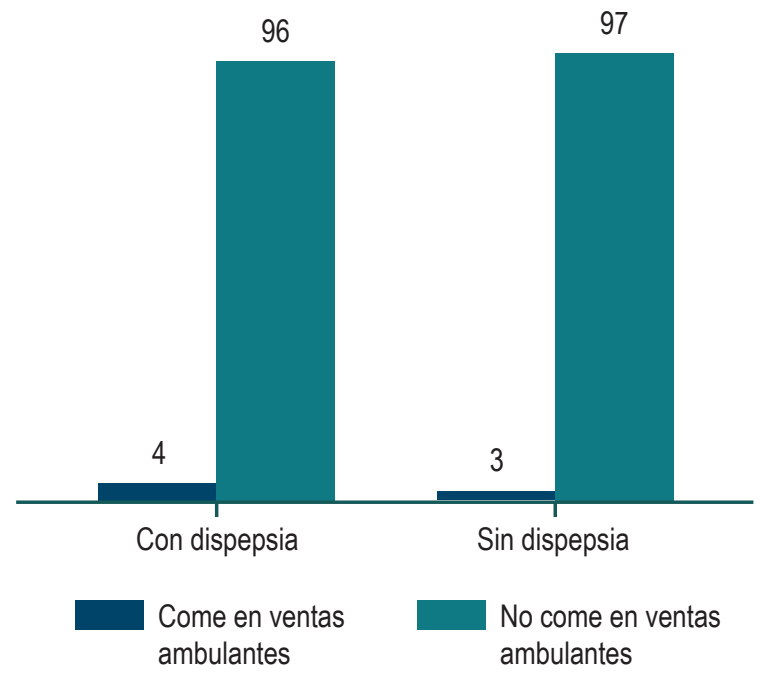

Figura 2. Dispepsia funcional según el consumo de alimentos en sitios de ventas ambulantes en estudiantes de medicina de siete departamentos del Perú.

Las mujeres tuvieron una asociación positiva al padecimiento de dispepsia funcional, y esto fue reportado por investigaciones locales ${ }^{(4,14,15)}$ e internacionales, en las que identificaron la asociación entre la sintomatología dispéptica y el sexo (valor $p=0,001)^{(16,17)}$.

Por último, tener horarios similares para la alimentación fue un factor asociado de manera negativa al padecimiento de dispepsia funcional. Esto ha sido reportado por algunos estudios $^{(9)}$, pero en otros no se encontró dicha asociación $^{(16)}$. Sin embargo, está relación no ha sido muy investigada en otras realidades, lo que puede ser un punto a considerar en próximas investigaciones.
Tabla 2. Análisis bivariado y multivariado de dispepsia funcional en estómago vacío.

\begin{tabular}{|lcccc|}
\hline \multicolumn{1}{l}{$\begin{array}{l}\text { Dispepsia } \\
\text { funcional }\end{array}$} & \multicolumn{3}{c}{ Bivariado } & \multicolumn{2}{c|}{ Multivariado } \\
\cline { 2 - 5 } & $\mathbf{R P c}($ IC 95 \%) & $\begin{array}{c}\text { Valor } \\
\mathbf{p}\end{array}$ & $\mathbf{R P a}($ IC 95 \%) & $\begin{array}{c}\text { Valor } \\
\mathbf{p}\end{array}$ \\
\hline $\begin{array}{l}\text { Come en ventas } \\
\text { ambulantes }\end{array}$ & $1,45(1,03-2,04)$ & 0,033 & $1,45(1,09-1,94)$ & 0,010 \\
\hline Mujeres & $1,43(1,15-1,78)$ & 0,001 & $1,40(1,15-1,71)$ & 0,001 \\
\hline Edad (años) & $0,97(0,93-1,02)$ & 0,256 & $0,98(0,95-1,02)$ & 0,395 \\
\hline Semestre* & $0,98(0,93-1,02)$ & 0,323 & $0,99(0,96-1,03)$ & 0,590 \\
\hline $\begin{array}{l}\text { Come en horarios } \\
\text { similares }\end{array}$ & $0,76(0,65-0,91)$ & 0,002 & $0,76(0,61-0,94)$ & 0,012 \\
\hline
\end{tabular}

$\mathrm{RPc}, \mathrm{RPa}$, IC $95 \%$ y valores $p$ obtenidos con modelos lineales generalizados, usando la familia Poisson, función de enlace log, modelos robustos y considerando la universidad sede como grupo clúster. *Tomados como variables cuantitativas.

Se tuvo la limitación de que los resultados obtenidos reflejan la realidad de solo siete departamentos del Perú, mas no de todos los estudiantes de medicina del Perú; sin embargo, los resultados son válidos por que se refleja esta realidad en siete de las ciudades más importantes del país. Por último, la potencia estadística fue la suficiente para determinar la asociación planteada.

Se concluye que quienes consumen sus alimentos en sitios de ventas ambulantes tienen una asociación positiva con el padecimiento de dispepsia funcional; además, otras variables influyentes en esta relación son el sexo femenino y que los estudiantes de medicina consuman sus alimentos en horarios similares.

\section{REFERENCIAS}

1. Godínez-Oviedo A. Prevalencia y causas de enfermedades gastrointestinales en niños del estado de Hidalgo, México. Salud Pública México. 2017;59(2):118-9. https://doi.org/10.21149/8064

2. Falcón MR, Barrón JM, Romero AL, Domínguez MF. Efecto adverso en la calidad proteica de los alimentos de dietas con alto contenido de fibra dietaria. Rev Chil Nutr. 2011;38(3):369-75. https://doi.org/10.4067/S0717-75182011000300012

3. Alejo Z, Rodríguez Fuente A, López Sosa D, Almaguer Sabina P. Patrón alimentario en adolescentes de octavo grado y su repercusión en la adecuación dietética. Medisur. 2011;9(6):518-22.

4. Vargas M, Talledo-Ulfe L, Samaniego RO, Heredia P, Rodríguez CA, Mogollón CA, Enríquez WF, Mejía CR. Dispepsia funcional en estudiantes de ocho facultades de medicina peruanas. Influencia de los hábitos. Acta Gastroenterológica Latinoam. 2016;46(2):95-101.

5. Kiani H, Haghighi A, Rostami A, Azargashb E, TABAEI SJS, Solgi A, Zebardast N. Prevalence, risk factors and symptoms associated to intestinal parasite infections among patients with gastrointestinal disorders in 
Nahavand, Western Iran. Rev Inst Med Trop São Paulo. 2016;58:42.

https://doi.org/10.1590/S1678-9946201658042

6. Campuzano S, Flórez DM, Ibarra CM, Sánchez PP.

Determinación de la calidad microbiológica y sanitaria de alimentos preparados vendidos en la vía pública de la ciudad de Bogotá D.C. Nova. 2015;13(23):81-92. https://doi.org/10.22490/24629448.1708

7. Cabanillas Torres G. Determinación de la presencia de Listeria Monocytogenes en quesos frescos artesanales provincia de Huarochirí, Lima-Perú. Lima: Universidad Ricardo Palma; 2019.

8. Bisbal-Murrugarra O, León-Barúa R, Berendson-Seminario $\mathrm{R}$, Biber-Poillevard M. A new questionnaire for the diagnosis of dyspepsia. Acta Gastroenterológica Latinoam. 2002;32(1):25-8.

9. Barker SF, Amoah P, Drechsel P. A probabilistic model of gastroenteritis risks associated with consumption of street food salads in Kumasi, Ghana: Evaluation of methods to estimate pathogen dose from water, produce or food quality. Sci Total Environ. 2014;487:130-142. https://doi.org/10.1016/j.scitotenv.2014.03.108

10. Nguyen VD, Sreenivasan N, Lam E, Ayers T, Kargbo D, Dafae F, Jambai A, Alemu W, Kamara A, Islam MS, Stroika S, Bopp C, Quick R, Mintz ED, Brunkard JM. Cholera epidemic associated with consumption of unsafe drinking water and street-vended water-eastern Freetown, Sierra Leone, 2012. Am J Trop Med Hyg. 2014;90(3):518-23. https://doi.org/10.4269/ajtmh.13-0567

11. Luquero FJ, Rondy M, Boncy J, Munger A, Mekaoui H, Rymshaw E, Page AL, Toure B, Degail MA, Nicolas S, Grandesso F, Ginsbourger M, Polonsky J, Alberti KP, Terzian M, Olson D, Porten K, Ciglenecki I. Mortality Rates during Cholera Epidemic, Haiti, 2010-2011. Emerg Infect Dis. 2016;22(3):410-6. https://doi.org/10.3201/eid2203.141970
12. Asiegbu CV, Lebelo SL, Tabit FT. The food safety knowledge and microbial hazards awareness of consumers of ready-to-eat street-vended food. Food Control. 2016;60:422-9. https://doi.org/10.1016/j.foodcont.2015.08.021

13. Sarker N, Islam S, Hasan M, Kabir F, Uddin MA, Noor R. Use of multiplex PCR assay for detection of diarrheagenic Escherichia coli in street vended food items. Am J Life Sci. 2013;1(6):267-72. https://doi.org/10.11648/j.ajls.20130106.15

14. Talledo-Ulfe L, Buitrago OD, Filorio Y, Casanova F, Campos L, Cortés F, Mejía CR. Factores asociados a dispepsia no investigada en estudiantes de 4 facultades de medicina de Latinoamérica: estudio multicéntrico. Rev Gastroenterol México. 2018;83(3):215-22. https://doi.org/10.1016/j.rgmx.2017.05.009

15. Vargas-Matos I, Ng-Sueng LF, Flores-Arriaga J, BeltránFlores S, Lema-Correa M, Piscoya A, Mayta-Tristán P. Superposición del síndrome de intestino irritable y dispepsia funcional basados en criterios ROMA III en estudiantes de medicina de una universidad privada de Lima, Perú. Rev Gastroenterol Perú. 2015;35(3):219-25.

16. Lee S-W, Lien H-C, Lee T-Y, Yang S-S, Yeh H-Z, Chang C-S. Etiologies of dyspepsia among a Chinese population: One hospital-based study. Open J Gastroenterol. 2014;4(06):249. https://doi.org/10.4236/ojgas.2014.46037

17. Yu J, Liu S, Fang XC, Zhang J, Gao J, Xiao YL, Zhu LM, Chen FR, Li ZS, Hu PJ, Ke MY, Hou XH. Gastrointestinal symptoms and associated factors in Chinese patients with functional dyspepsia. World J Gastroenterol. 2013;19(32):5357-64. https://doi.org/10.3748/wjg.v19.i32.5357 